\title{
Strong spin orientation-dependent spin current diffusion and inverse spin Hall effect in a ferromagnetic metal
}

\author{
Zhaozhao Zhu ${ }^{1,2}$, Xiaoli Zheng ${ }^{1,3}$, Gang $\mathrm{Li}^{1,2}$, He Bai ${ }^{1,2}$, Jian Su ${ }^{1,2}$, Ying Zhang ${ }^{1}$ and Jian-Wang Cai ${ }^{1,2}$
}

\begin{abstract}
Pure spin current transport has become the central point of the state-of-the-art spintronics. While most spin current phenomena have been extensively explored, aspects of the pure spin current injected into ferromagnetic metals are far from completely understood. The reports on a fundamental problem, i.e. the spin relaxation asymmetry with spin current polarization collinear or transverse to the magnetization of ferromagnetic metals, are quite controversial. By employing a $\mathrm{Y}_{3} \mathrm{Fe}_{5} \mathrm{O}_{12}(\mathrm{YIG}) / \mathrm{Cu} / \mathrm{Ni}_{80} \mathrm{Fe}_{20}(\mathrm{Py}) / \mathrm{Ir}_{25} \mathrm{Mnn}_{75}$ (IrMn) spin valve heterostructure with the thermal inverse spin Hall effect (ISHE) of a Py well separated from other thermoelectric transport and thermal Hall effects, we find that the ISHE signal amplitude in $10 \mathrm{~nm}$ Py increases by $80 \%$ when changing the relative orientation of the YIG and Py magnetization from orthogonal $(\perp)$ to collinear $(\|)$. Moreover, the spin-diffusion length $\lambda_{\mathrm{sf}}$ and effective spin Hall angle $\theta_{\mathrm{SH}}^{\text {eff }}$ of Py are also spin orientation dependent and vary from $\lambda_{\mathrm{sf}}^{\perp}=1.0 \pm 0.1 \mathrm{~nm}$ to $\lambda_{\mathrm{sf}}^{\|}=2.8 \pm 0.5 \mathrm{~nm}$ with $\theta_{\mathrm{SH}}^{\text {eff }}(\perp) / \theta_{\mathrm{SH}}^{\text {eff }}(\|)=1.5$, respectively. Our results demonstrate magnetization orientation-dependent spin relaxation and spin injection efficiency of a pure spin current, revealing that exchange interactions in ferromagnetic metals strongly affect the transport of the pure spin current.
\end{abstract}

\section{Introduction}

In past years, pure spin current has received considerable interest in spintronics due to its non-Joule heat properties. The ferromagnetic insulator (FMI)/nonmagnetic metal (NM) bilayer is one of the most common structures for studying pure spin current phenomena, where FMI represented by $\mathrm{Y}_{3} \mathrm{Fe}_{5} \mathrm{O}_{12}$ (YIG) can generate pure spin current through magnetization precession or a temperature gradient while heavy NMs such as Pt with strong spin-orbit coupling (SOC) can serve as spin current detectors via the inverse spin Hall effect (ISHE) $)^{1-3}$. When a temperature gradient is applied perpendicular to the YIG/NM interface, a magnon spin current can be

\footnotetext{
Correspondence: Jian-Wang Cai (jwcai@iphy.ac.cn)

${ }^{1}$ Beijing National Laboratory for Condensed Matter, Institute of Physics,

Chinese Academy of Sciences, Beijing 100190, People's Republic of China

${ }^{2}$ School of Physical Sciences, University of Chinese Academy of Sciences,

Beijing 100049, People's Republic of China

Full list of author information is available at the end of the article.

These authors contributed equally: Zhaozhao Zhu, Xiaoli Zheng
}

created across the whole FMI and then pumped into the $\mathrm{NM}$ layer because of the magnon accumulation at the YIG/NM interface, and the injected spin current in the NM is finally transformed into an electric voltage. This is the so-called longitudinal spin-Seebeck effect $(\mathrm{LSSE})^{3,4}$. Although the validity of the SSE has been challenged by the parasitic thermal electric effect and thermal Hall effect, such as the anomalous Nernst effect $(\mathrm{ANE})^{4-6}$, magnon Hall effect $(\mathrm{MHE})^{7,8}$ and anomalous Righi-Leduc effect (ARLE) $)^{9,10}$, it has been verified in many YIG-based heterostructures that LSSE remains a reliable methodology to investigate pure spin current phenomena in the framework of spin caloritronics ${ }^{5,11}$.

Ferromagnetic metals (FMMs) have also been found to exhibit a significant ISHE ${ }^{12}$, and by means of $\operatorname{LSSE}^{12-15}$, spin pumping ${ }^{16,17}$ and the nonlocal electrical injection and detection method ${ }^{18-20}$, the ISHE in $\mathrm{Fe}, \mathrm{Co}, \mathrm{Ni}$ and their alloys has been systematically investigated recently. Because of the presence of spin polarized electrons and 
spontaneous magnetization, spin relaxation in FMMs is actually much more complex than that in NMs. Early magnetotransport ${ }^{21,22}$ and ferromagnetic resonance $(\mathrm{FMR})^{23,24}$ experiments showed that longitudinal and transverse spin relaxation in FMMs are governed by different mechanisms, corresponding to two different spin relaxation distances when the spin polarization $\vec{\sigma}$ of the injected current (or spin current) is collinear or transverse to the magnetization of ferromagnetic metals. The ISHE can be expressed as $\vec{J}_{\mathrm{c}} \propto \theta_{\mathrm{SH}} \vec{J}_{\mathrm{s}} \times \vec{\sigma}$, where $\theta_{\mathrm{SH}}$ is the spin Hall angle; $\vec{J}_{\mathrm{c}}$ and $\vec{J}_{\mathrm{s}}$ represent the charge current and spin current, respectively; and $\vec{\sigma}$ is the polarization direction of the spin current. The variation in $\vec{J}_{\mathrm{s}}$ in a material is governed by its relaxation mechanism. Thus, the ISHE of FMMs can be used as a tool to study the mechanism of spin relaxation of pure spin current in FMMs. Surprisingly, Tian et al. ${ }^{25}$ and Li et al. ${ }^{26}$ found that the ISHE and spin relaxation of Co are irrelevant to its magnetization direction in LSSE and FMR-spin pumping experiments, respectively. A recent study on magnon transport in a nonlocal YIG/Py hybrid structure reported that there exists a transverse spin accumulation orthogonal to the direction of the applied charge current $(\vec{I})$ and magnetization $(\vec{M})$ in Py. This magnetization-dependent charge/spin conversion in Py is termed the anomalous spin Hall effect (ASHE) and is assumed to have the same spin relaxation distance as the ordinary SHE/ISHE with negligible spin dephasing in $\mathrm{Py}^{18}$. These controversial results reflect that the effect of magnetization on the pure spin current in FMMs, one of the most fundamental issues in spintronics, remains unsettled. Parenthetically, it is important to mention that the magnetizationdependent ISHE or ASHE is different from the anisotropic spin Hall effect in nonmagnetic metals ${ }^{27}$ and antiferromagnetic metals ${ }^{28,29}$, as well as the anisotropic absorption of pure spin current in pseudo spin valve heterostructures ${ }^{30}$, where the relevant anisotropy originates from the interaction between the spin current polarization $\vec{\sigma}$ and specific crystallographic symmetries ${ }^{27}$ or antiferromagnetic spin configurations with respect to the crystallographic axis ${ }^{28,29}$.

In this article, we demonstrate that the ISHE and relaxation of pure spin current in a ferromagnetic metallic permalloy $\left(\mathrm{Ni}_{80} \mathrm{Fe}_{20}\right.$, denoted as Py) are strongly magnetization orientation dependent. By means of LSSE measurements on a YIG/Cu/Py/IrMn spin valve structure, where the spin index of the spin current is determined by the direction of YIG magnetization $\vec{M}_{\mathrm{YIG}}$, the magnetization of Py $\vec{M}_{\mathrm{Py}}$ is pinned by the antiferromagnetic (AFM) IrMn. We observed an appreciable increase in the thermal ISHE voltage of Py of up to $80 \%$ when the relative orientation $\vec{M}_{\mathrm{YIG}}, \vec{M}_{\mathrm{Py}}$ changed from orthogonal $(\perp)$ to collinear $(\|)$. By systematically varying the Py thickness, we determined the spin orientationdependent spin diffusion length $\lambda_{\mathrm{sf}}^{\perp}, \lambda_{\mathrm{sf}}^{\|}$and the ratio of effective spin Hall angle $\theta_{\mathrm{SH}}^{\text {eff }}=J_{\mathrm{s}}(0) \theta_{\mathrm{SH}}$, i.e., $\lambda_{\mathrm{sf}}^{\perp}=1.0 \pm$ $0.1 \mathrm{~nm}, \lambda_{\mathrm{sf}}^{\|}=2.8 \pm 0.5 \mathrm{~nm}$, and $\theta_{\mathrm{SH}}^{\text {eff }}(\perp) / \theta_{\mathrm{SH}}^{\text {eff }}(\|)=1.5$. The present results show that the magnetization of a ferromagnetic metal greatly affects the spin relaxation process and interfacial spin injection efficiency of the spin current simultaneously.

\section{Materials and methods \\ Sample preparation}

The YIG films with thicknesses of $100 \mathrm{~nm}$ were deposited on $6 \mathrm{~mm} \times 6 \mathrm{~mm} \times 0.5 \mathrm{~mm} \mathrm{Gd}_{3} \mathrm{Ga}_{5} \mathrm{O}_{12}(\mathrm{GGG})$ single crystal substrate with (111) orientation by off-axis magnetron sputtering at room temperature. The base pressure of the sputtering system was better than $4 \times$ $10^{-6} \mathrm{~Pa}$, and the working gas was high-purity $(5 \mathrm{~N}) \mathrm{Ar}$ at a pressure of $1.0 \mathrm{~Pa}$. The sputtering rate was approximately $0.029 \mathrm{~nm} / \mathrm{s}$ with an RF power of $50 \mathrm{~W}$. Samples were subsequently annealed at $800^{\circ} \mathrm{C}$ for $2 \mathrm{~h}$ in a quartz tube with a pure oxygen pressure of $450 \mathrm{~Pa}$ under an oxygen flow rate of 45 SCCM. Radial pattern shadow masks composed of eight $0.3 \mathrm{~mm} \times 5.6 \mathrm{~mm}$ strip hollows were placed on the YIG films to grow the metallic multilayers by on-axis dc magnetron sputtering, and a static field of approximately 280 Oe was applied during sputtering to induce exchange bias in the Py/IrMn structure.

\section{Magnetization and thermal voltage measurements}

The magnetization of the YIG and metallic films was measured using a vibrating sample magnetometer (VSM, Model 4 HF, MicroSense LLC) at room temperature. The LSSE of the YIG-based spin valve was investigated in a customized magnetotransport measurement system with an electromagnet strength as high as $4 \mathrm{kOe}$. For the LSSE measurement with a temperature gradient $\nabla T_{z}$ normal to the film plane, the samples (measuring $6 \times 6 \times 0.5 \mathrm{~mm}^{3}$ ) are placed between two $\mathrm{Cu}$ blocks at two different temperatures, which are stacked symmetrically along the vertical central axis ( $z$ axis). To ensure heat contact with the two $\mathrm{Cu}$ blocks and samples, a thermal conductive silicon pad is taped to the surfaces of the blocks. The bottom $\mathrm{Cu}$ block, which is shaped as a cylinder with a height of $125 \mathrm{~mm}$ and a diameter of $30 \mathrm{~mm}$, is placed on a horizontally rotatable stage. The top $\mathrm{Cu}$ block is rectangular in shape with dimensions $13 \times 9 \times 1.5 \mathrm{~mm}^{3}$ and is fixed on a circular printed circuit board (PCB) $(26 \mathrm{~mm}$ in diameter). A Pt $100-\Omega$ heater $\left(5 \times 2 \times 1 \mathrm{~mm}^{3}\right)$ (heat source) is fixed at the center of the $\mathrm{Cu}$ block back using silver paint, which allows the central part of the top $\mathrm{Cu}$ block surface to be quite uniformly heated over a large area $\left(6 \times 6 \mathrm{~mm}^{2}\right.$ at least). The temperature difference between the bottom of the substrate and the top of the 
film is approximately $13 \mathrm{~K}$ for the LSSE measurements as determined by two thermocouples.

\section{Results}

Magnetic characterization and experimental configuration

The multilayer films studied have the structure GGG/ YIG $(100 \mathrm{~nm}) / \mathrm{Cu}(3.5 \mathrm{~nm}) / \mathrm{Py}\left(t_{\mathrm{Py}} \mathrm{nm}\right) / \mathrm{IrMn}(8 \mathrm{~nm}) / \mathrm{SiO}_{2}$ $(5 \mathrm{~nm})$ with a Py thickness $\left(t_{\mathrm{Py}}\right)$ ranging from 3 to $12 \mathrm{~nm}$. The top $\mathrm{SiO}_{2}$ layer is used to protect the metallic layers from oxidation. The $\mathrm{Cu}$ spacer is fixed at $3.5 \mathrm{~nm}$ to avoid interlayer coupling between the YIG and Py, and the samples exhibit an appreciable thermal voltage with a limited shunting effect. The high-quality epitaxial YIG films used are magnetically soft and isotropic in the film plane with a very small saturation field $\left(H_{\mathrm{s}}<3 \mathrm{Oe}\right)^{31}$. The samples are magnetically characterized using VSM. Figure 1a, b displays the hysteresis loops of a patterned sample $\left(t_{\mathrm{Py}}=10 \mathrm{~nm}\right)$ with the in-plane field applied parallel and orthogonal to the exchange bias direction, respectively. One notes that $\vec{M}_{\mathrm{YIG}}$ is switched at a field smaller than 3 Oe regardless of the external field direction, whereas $\vec{M}_{\text {Py }}$ shows a shifted loop centered at -200 Oe when the field is parallel to the exchange bias direction or slowly rotates to the field direction to a limit of $\pm(200-300)$ Oe when the field is perpendicular to the exchange bias direction. It is reasonable to say that $\vec{M}_{\text {Py }}$ is fixed at the exchange bias direction, while the YIG magnetization is aligned to different in-plane directions by a
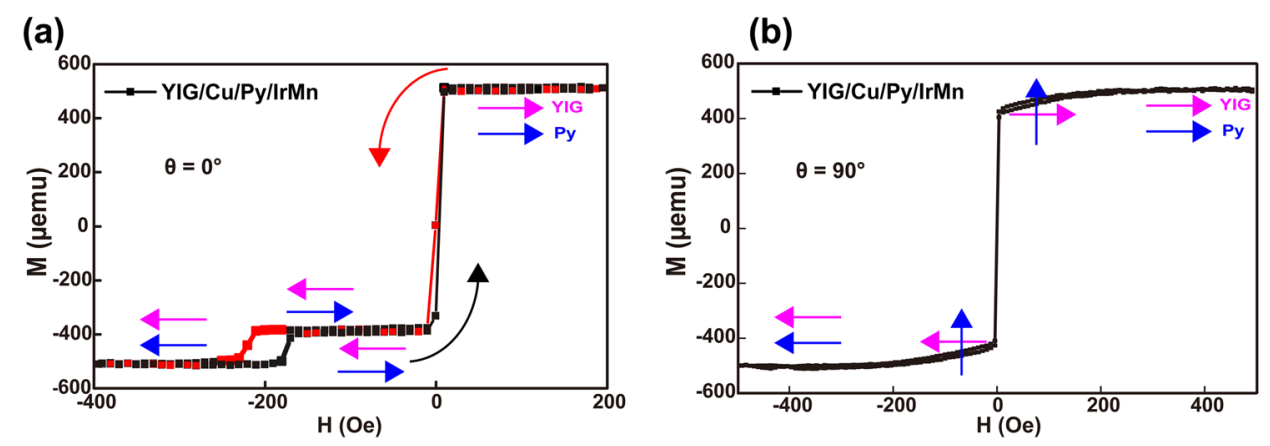

(c)

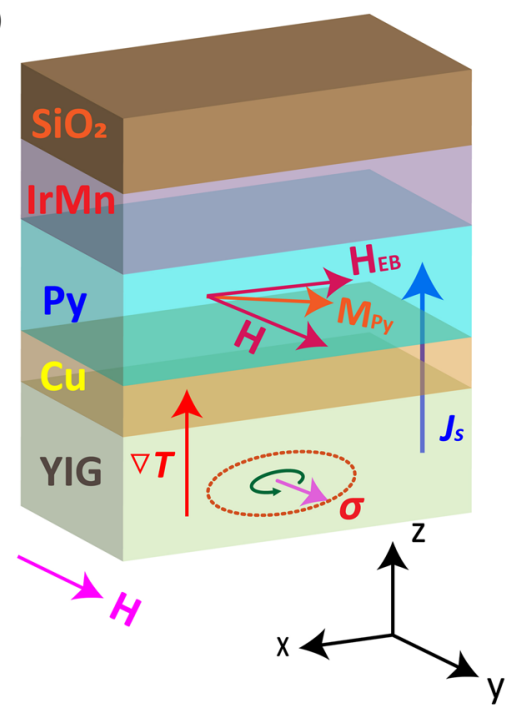

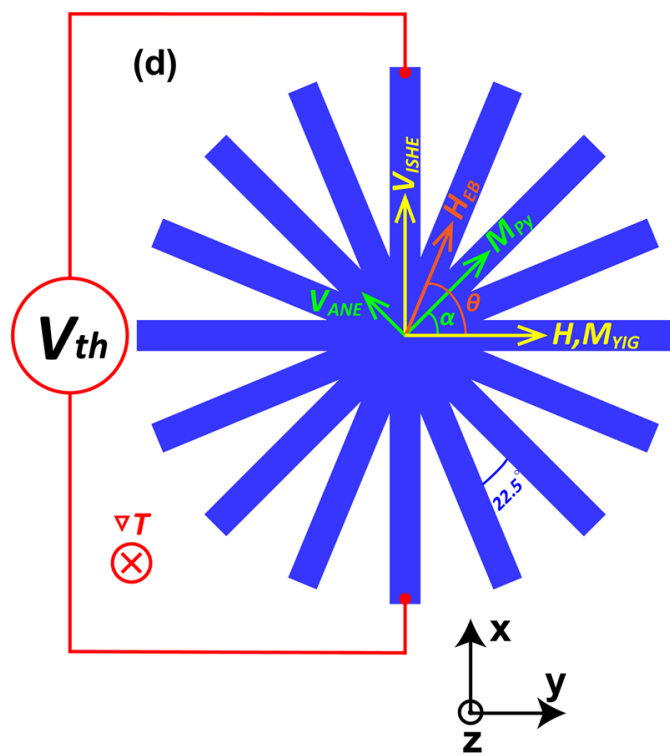

Fig. $\mathbf{M}-\boldsymbol{H}$ characterization and experimental setup. $M-H$ loops of the patterned sample $\mathrm{YIG}(100 \mathrm{~nm}) / \mathrm{Cu}(3.5 \mathrm{~nm}) / \mathrm{Py}(10 \mathrm{~nm}) / / \mathrm{rMn}(8 \mathrm{~nm}) / \mathrm{SiO}{ }_{2}$ $(5 \mathrm{~nm})$ with a magnetic field applied $\mathbf{a}$ parallel and $\mathbf{b}$ orthogonal to the exchange bias direction. Schematic diagrams of $\mathbf{c}$ the multilayer structure and experiment configuration and $\mathbf{d}$ the coordinate and azimuth for magnetization orientation-dependent LSSE measurement. The metallic layers of samples are radially patterned with eight strip channels (relative alignment of $22.5^{\circ}$ ), the temperature gradient (on the $z$ axis) is applied perpendicularly to the film plane (on the $x-y$ plane), the external field $\vec{H}$ is applied along the $y$ axis, which will align the magnetization of YIG $\left(\vec{M}_{\mathrm{YIG}}\right)$ and the spin polarization $(\vec{\sigma})$ of the spin current, and the thermal voltage is measured through the channel on the $x$ axis by the two end electrodes. Here, $\theta$ means the angle between $\vec{H}$ and the exchange-bias direction $\left(\vec{H}_{\mathrm{EB}}\right)$, changing for the different voltage measurement channels; $a$ represents the direction of the magnetization of Py $\left(\vec{M}_{\mathrm{Py}}\right)$ with respect to $\vec{H}$. It is approximately taken that $a=\theta$ when the YIG magnetization is aligned by a small field. 
small external field (e.g., 3 Oe). This also holds for all other samples with different Py thicknesses because the exchange bias field of all samples exceeds 150 Oe in this study.

The schematic illustration of the multilayer structure and the angular ISHE measurement method is detailed in Fig. 1c, d. A temperature gradient $\nabla T=13 \mathrm{~K}$ between the sample surface and substrate bottom is applied along the film normal $(z \text { axis })^{13,32}$, which can produce spin accumulation between the YIG and the metallic multilayers. The resultant spin current can pass through the $\mathrm{Cu}$ layer and be absorbed by the Py/IrMn structure. It should be pointed out that the FMM/AFM exchange bias structure has been proven to be an efficient spin sink structure ${ }^{13,33}$. More importantly, when a small external field $\vec{H}$ (e.g., $3 \mathrm{Oe})$ that exceeds the YIG saturation field is applied along the $y$ axis, $\vec{M}_{\text {YIG }}$ and the spin polarization $\vec{\sigma}$ of spin current are also aligned along the $y$ axis; then, one can obtain the ISHE voltage $V_{\text {ISHE }}$ by measuring the corresponding voltage in the strip along the $x$ axis according to $\vec{E}_{\text {ISHE }} \propto \vec{J}_{s} \times \vec{\sigma}$. As will be proven below, other thermal voltages entangled with $V_{\text {ISHE }}$ are negligible because $\vec{M}_{\mathrm{Py}}$ is fixed in the exchange bias direction during the YIG magnetization reversal at the small field. Under the same measurement conditions, the different strips of the same sample arranged in the $x$ axis will give the corresponding $V_{\text {ISHE }}$ of Py with different magnetization orientations.

\section{Magnetization orientation-modulated ISHE voltage in Py}

Before presenting thermal ISHE measurement results, we would like to outline all possible contributions to the thermal voltage in a spin valve heterostructure comprising an FM insulator (YIG) and a ferromagnetic metal (Py) exchange biased by an AFM (IrMn) with the LSSE configuration. These include contributions not only from the ISHE but also from ANE and ARLE ${ }^{11}$ and can be described by the following formulas:

$$
\begin{aligned}
& \vec{V}_{\mathrm{ISHE}} \propto \theta_{\mathrm{SH}} \vec{J}_{\mathrm{s}} \times \vec{\sigma}, \\
& \vec{V}_{\mathrm{ANE}} \propto-S_{x y} \vec{\nabla} T \times \vec{M}, \\
& \vec{V}_{\mathrm{ARLE}} \propto \Delta \vec{J}_{Q} \times \vec{M},
\end{aligned}
$$

where $\vec{\sigma}$ is the polarization direction of the injected spin current $\vec{J}_{\mathrm{s}}, S_{x y}$ is the transverse Seebeck coefficient, $\vec{M}$ is the magnetization vector of the FMM; $\Delta S$ is the difference in the Seebeck coefficients of the metallic multilayers and contacting wires, and $\vec{J}_{Q}$ is the heat current in the FMM caused by the temperature gradient $\nabla T$. By sweeping the external field, the $\vec{V}_{\text {ISHE }}$ signal of the Py layer generated by the spin current from YIG will vary in response to $\vec{M}_{\text {YIG }}$, whereas both $\vec{V}_{\text {ANE }}$ and $\vec{V}_{\text {ARLE }}$ signals will vary in response to $\vec{M}_{\mathrm{Py}}$. It should be pointed out that in the spin valve heterostructure, an ISHE voltage signal in IrMn
( $\left.V_{\text {ISHE@AFM }}\right)$ will also be induced due to the injected spin current produced by Py, but this signal is irrelevant to $V_{\text {ISHE }}$ of the Py and varies in response to $\vec{M}_{\mathrm{Py}}$. For simplicity, we hereafter refer $\vec{V}_{\mathrm{ANE}-\text { Like }}$ for all thermal voltages in response to $\vec{M}_{\mathrm{Py}}$, including conventional $\vec{V}_{\mathrm{ANE}}$, $\vec{V}_{\text {ARLE }}$ and $\vec{V}_{\text {ISHE@AFM }}$.

Figure 2a shows the thermal voltage loop of the sample YIG/Cu/Py $(10 \mathrm{~nm}) / \mathrm{IrMn}$ with an external field parallel to the exchange bias direction $\left(\theta=0^{\circ}\right)$. The well-separated magnetization reversals of YIG and Py displayed in Fig. 1a are also reflected in the thermal voltage loop except that the thermo-magnetic-electric measurement gives a slightly smaller exchange bias field $\left(H_{\mathrm{EB}} \approx 160 \mathrm{Oe}\right)$. This can be attributed to the sample temperature increase when the temperature gradient is exerted. From Fig. 2a, we directly obtain $V_{\mathrm{ISHE}}=0.22 \mu \mathrm{V}$ at $\vec{M}_{\mathrm{Py}} \| \vec{\sigma}\left(M_{\mathrm{YIG}}\right)$ and $V_{\mathrm{ANE}-\mathrm{Like}}=0.64 \mu \mathrm{V}$, corresponding to the respective thermal voltage related to the YIG magnetization reversal within \pm 3 Oe and the shifted subloop of Py. When the external field is applied perpendicular to the exchange bias direction $\left(\theta=90^{\circ}\right)$, the thermal voltage loop displayed in Fig. 2b for the same sample also resembles its $M-H$ counterpart as expected. Since YIG is extremely soft, with $H_{\mathrm{S}}$ below one fiftieth of the Py pinning field $H_{\mathrm{EB}}$, we determine $V_{\text {ISHE }}$ at any $\theta$ angle as the thermal voltage difference at the fields of \pm 3 Oe, within which YIG magnetization reversal is just completed, whereas $\vec{M}_{\mathrm{Py}}$ is almost unchanged with negligible $V_{\text {ANE-Like }}$ involved. We thus obtain $V_{\mathrm{ISHE}}=0.12 \mu \mathrm{V}$ at $\vec{M}_{\mathrm{Py} \perp} \vec{\sigma}\left(M_{\mathrm{YIG}}\right)$, which is significantly smaller than the value at $\vec{M}_{\mathrm{Py}} \mid \vec{\sigma}\left(M_{\mathrm{YIG}}\right)$. The extended ANE voltage (i.e., $V_{\text {ANE-Like }}$ ) is acquired by subtracting $V_{\text {ISHE }}$ from the saturated total thermal voltage, which is approximately $0.73 \mu \mathrm{V}$, which is also quite different from the value for the parallel field case. We would like to emphasize that within the YIG switching fields of $\pm 3 \mathrm{Oe}$, the change of $V_{\text {ANE-Like }}$ is estimated to be less than $0.02 \mu \mathrm{V}$, indeed far smaller than $V_{\text {ISHE, proving }}$ that the acquisition of $V_{\mathrm{ISHE}}$ and $V_{\mathrm{ANE}-L i k e}$ by the above method is reliable in the present spin valve heterostructure. To rule out other possible artifacts caused by the experiment, we also measured the ISHE voltage of a YIG $(100 \mathrm{~nm}) / \mathrm{Pt}(3 \mathrm{~nm})$ sample under the same conditions. The thermal voltages of the eight Pt strips are all saturated within 3 Oe, showing that the magnetism of YIG is extremely soft and isotropic. We observed ISHE voltage fluctuations for different Pt strips, but the variation was less than $5 \%$ of the average amplitude, which seems to be caused by the tiny variation of the electrode distance due to the location error of the bonding points on the strip terminals in different measurements (for more details, see Supplementary Fig. S1a, b). We also prepared a GGG/Cu $(3.5 \mathrm{~nm}) / \mathrm{Py}(10 \mathrm{~nm}) / \mathrm{IrMn}(8 \mathrm{~nm}) / \mathrm{SiO}_{2}(5 \mathrm{~nm})$ control sample, and we note that the saturated thermal voltage is independent of the exchange bias direction except for a 

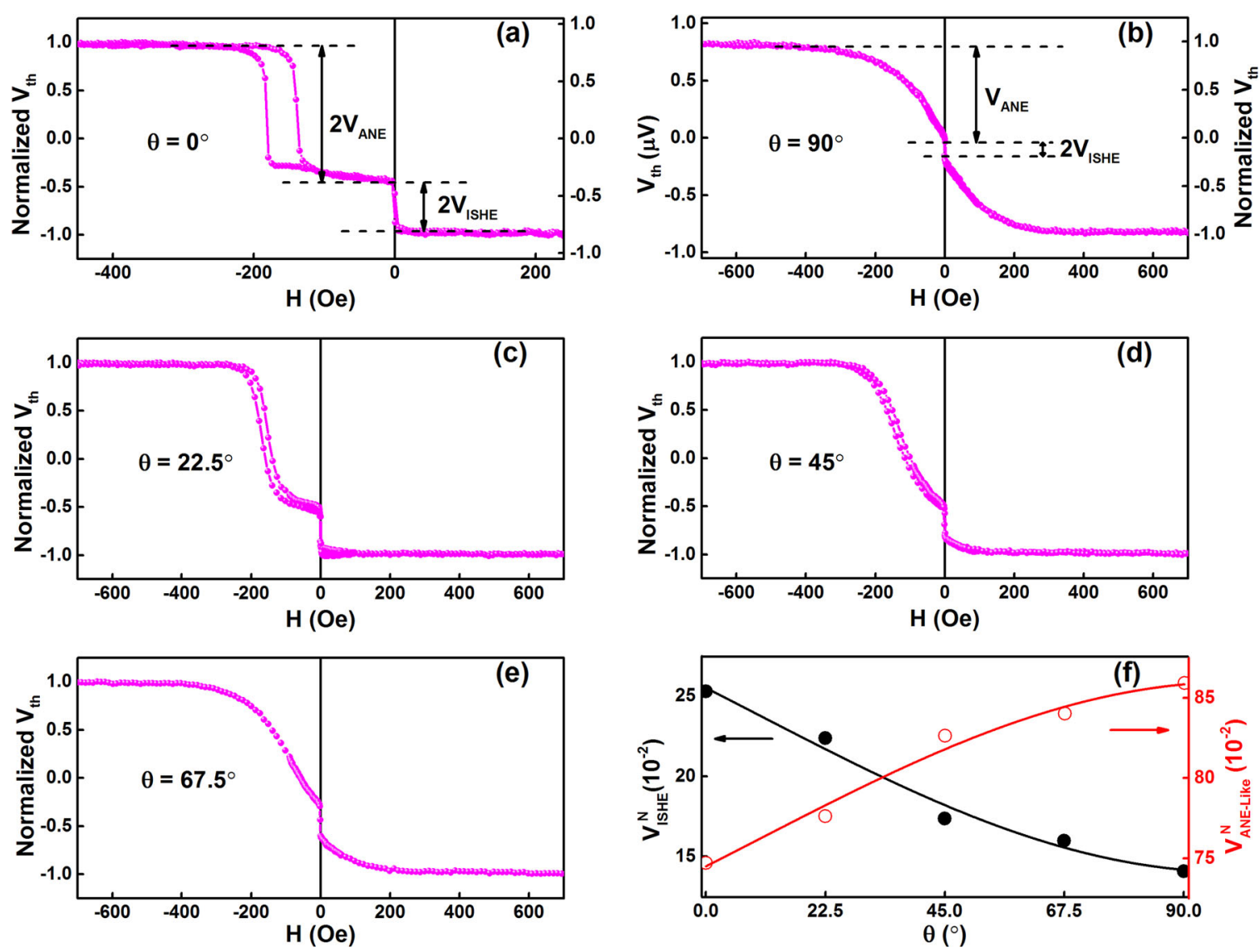

Fig. 2 Variation in ISHE and ANE-Like signal at different $\boldsymbol{\theta}$. a-e Thermal voltage $V_{\text {th }}$ Vs. $H_{\text {, where }} V_{\text {th }}$ in the channel on the $x$ axis is recorded while field sweeps along the $y$ axis. In the magnetization reversal process of YIG ( \pm 3 Oe), the Py magnetization is fixed by a large exchange field $\vec{H}_{\mathrm{EB}}$ at an angle $\theta$ with respect to the external field $\vec{H}$. $\mathbf{f}$ The $\theta$ dependence of the fraction of $V_{\text {ISHE }}$ and $V_{\text {ANE-Like }}$ (denoted by $V_{\text {ISHE }}^{N}$ and $V_{\text {ANE-Like' }}^{N}$ respectively) in the total thermal voltage, where the red and black curves are fit using a sine function.

random small fluctuation (below 5\%) for the eight strips (see Supplementary Fig. S4). It should be pointed out that the spin Hall angle of IrMn is demonstrated to be independent of the exchange bias direction by spin-torque ferromagnetic resonance (ST-FMR) measurements on Py/ $\mathrm{IrMn}^{34}$. Therefore, the ISHE voltage in Py is intrinsically modified significantly when the Py magnetization is changed from the parallel to the perpendicular direction with respect to the polarization direction of the injected spin current. Parenthetically, the introduction of pure spin current into the Py layer causes an additional contribution to $V_{\mathrm{ANE}-L i k e}$ as well, and this contribution also strongly depends on the polarization direction of the pure spin current $\left(\vec{M}_{\mathrm{YIG}}\right)$ with respect to $\vec{M}_{\mathrm{Py}}$, although $\vec{M}_{\mathrm{Py}}$ itself is unvaried from the magnetostatic point of view.

The saturated total thermal voltage in the present spin valve heterostructure can be expressed as $V_{\mathrm{th}}^{\text {thal }}=$ $\left|2 V_{\mathrm{ISHE}}\left(\vec{M}_{\mathrm{Py}}|| \vec{M}_{\mathrm{YIG}}(\vec{\sigma})|| \vec{H}_{\text {high }}\right)\right|+\mid 2 V_{\mathrm{ANE}-\text { Like }}\left(\vec{M}_{\mathrm{Py}}|| \vec{M}_{\mathrm{YIG}}\right.$ $\left.(\vec{\sigma})|| \vec{H}_{\text {high }}\right) \mid$. According to Eqs. (1)-(3), this voltage should take the same value for the same sample regardless of the intermediate magnetic configurations caused by the different field directions. To minimize the experimental error in the evaluation of the $\theta$ dependence of $V_{\text {ISHE }}$ and $V_{\text {ANE-Like, the thermal voltage as a function of the external }}$ field is normalized to its saturated total thermal voltage for the sample YIG/Cu/Py $(10 \mathrm{~nm}) / \mathrm{IrMn}$ at different $\theta$. The rescaled results are compared in Fig. 2a-e. We extract the data $V_{\mathrm{ISHE}}^{N}=\frac{V_{\text {ISHE }}}{V_{\text {thal }}^{\text {total }}}$ from Fig. 2a-e and plot $V_{\text {ISHE }}^{N}$ as a function of $\theta$ in Fig. 2f. The fraction of $V_{\text {ISHE }}$ in the total thermal voltage $V_{\text {th }}^{\text {total }}$ decreases from 0.25 to 0.14 with $\theta$ increasing from $0^{\circ}$ to $90^{\circ}$ and shows a sine function dependence on $\theta$. This reflects that the transport of the injected spin current in Py is greatly affected by the Py magnetization because of the strong exchange interaction between the injected spins and the $3 \mathrm{~d}$ moment in Py. We would like to point out that the fraction of $V_{\mathrm{ANE}}$ Like $\left(V_{\mathrm{ANE}-\text { Like }}^{N}\right)$ in the total thermal voltage, $V_{\mathrm{ANE}-\text { Like }}^{N}=$ $\frac{V_{\text {ANE-Like }}}{V_{\text {thal }}^{\text {total }}}=1-V_{\text {ISHE }}^{N}$ as also shown in Fig. $2 \mathrm{f}$, increases from 0.75 to 0.86 when $\theta$ varies from $0^{\circ}$ to $90^{\circ}$. We have 
further comparatively studied the ANE-Like signals in GGG/Cu $(3.5 \mathrm{~nm}) / \mathrm{Py}(10 \mathrm{~nm}) / \mathrm{IrMn}$, and there is no recognizable variation in $V_{\text {ANE-Like }}$ (for more details, see the Supplementary Information Section D). The ANELike voltage is indeed modified in the spin-valve heterostructure due to the injected spin current with different spin polarization directions, indicative of a new approach to manipulate the spin-dependent transport in a ferromagnetic metal.

Other samples with different Py thicknesses show a similar angular dependence of $V_{\text {ISHE}}^{N}$, but the difference in $V_{\text {ISHE }}^{N}$ between $\theta=0^{\circ}$ and $90^{\circ}$ is changed. To quantitatively describe the spin orientation-dependent ISHE, we define the ratio of the angular $V_{\mathrm{ISHE}}$ difference as $\operatorname{Ra}\left(V_{\mathrm{ISHE}}\right)=\frac{\Delta V_{\text {ISHE }}}{V_{\mathrm{ISHE}}\left(90^{\circ}\right)}=\frac{\left|V_{\text {ISHE }}^{N}\left(0^{\circ}\right)-V_{\text {ISHE }}^{N}\left(90^{\circ}\right)\right|}{V_{\text {ISHE }}^{N}\left(90^{\circ}\right)}$. Figure 3 shows the $\mathrm{Ra}\left(V_{\text {ISHE }}\right)$ results of all YIG $(100 \mathrm{~nm}) / \mathrm{Cu}(3.5 \mathrm{~nm}) / \mathrm{Py}$ $(3-12 \mathrm{~nm}) / \operatorname{IrMn}(8 \mathrm{~nm})$ samples. One notes that $\frac{\Delta V_{\text {ISHE }}}{V_{\text {ISHE }}\left(90^{\circ}\right)}$ increases appreciably with increasing $t_{\mathrm{Py}}$ and reaches a maximum of $80 \%$ at $t_{\mathrm{Py}}=10 \mathrm{~nm}$. Assuming that $t_{\mathrm{Py}}$ is much larger than the spin diffusion length $\left(\lambda_{\mathrm{sf}}\right)$ of Py, which is satisfied as we will prove later, $V_{\mathrm{ISHE}}$ generated in Py will vary in proportion to its resistance and thus approximately in proportion to the inverse of $t_{\mathrm{Py}}$. The increasing tendency of $\frac{\Delta V_{\mathrm{ISHE}}}{V_{\mathrm{ISHE}}\left(90^{\circ}\right)}$ with increasing $t_{\mathrm{Py}}$ suggests that the decay rate of $\Delta V_{\text {ISHE }}$ caused by $\theta$ is slow than the decrease in $V_{\text {ISHE. Importantly, the large } \mathrm{Ra}}$ $\left(V_{\mathrm{ISHE}}\right)$ is observed throughout the $t_{\mathrm{Py}}$ range studied here, indicating that the ISHE voltage is strongly modulated by spin orientation on the nanometer scale. We would like to emphasize that because of the anisotropic magnetoresistance (AMR) for metallic ferromagnets, the resistance of the YIG/Cu/Py/IrMn strips at different $\theta$ should be different during the YIG reversal process with Py pinned in different directions, which will accordingly alter the corresponding measured thermal voltage. However, AMR in thin Py films at room temperature is rather small, below $\sim 1 \%$ for Py films with thicknesses less than $10 \mathrm{~nm}^{35}$. In fact, $\quad \mathrm{AMR} \approx 0.1-0.3 \%$ for $\mathrm{GGG} / \mathrm{Cu} \quad(3.5 \mathrm{~nm}) / \mathrm{Py}$ $(5-10 \mathrm{~nm}) / \mathrm{IrMn}(8 \mathrm{~nm}) / \mathrm{SiO}_{2}(5 \mathrm{~nm})$ due to the shunting effect of $\mathrm{Cu}$ and IrMn layers (for more details, see Supplementary Fig. S2). Therefore, the contribution of AMR to the observed angular-dependent ISHE should be negligible.

\section{Magnetization orientation-dependent spin diffusion length and effective spin Hall angle in Py}

Below, we focus on the mechanism of the magnetization orientation affecting the transport of pure spin current in ferromagnetic metals. We calculate the absolute magnitude of the thermal ISHE voltage generated in Py (denoted as $V_{\text {ISHE }}^{\mathrm{Py}}$ ) at different thicknesses by subtracting the shunting effect of the other conducting

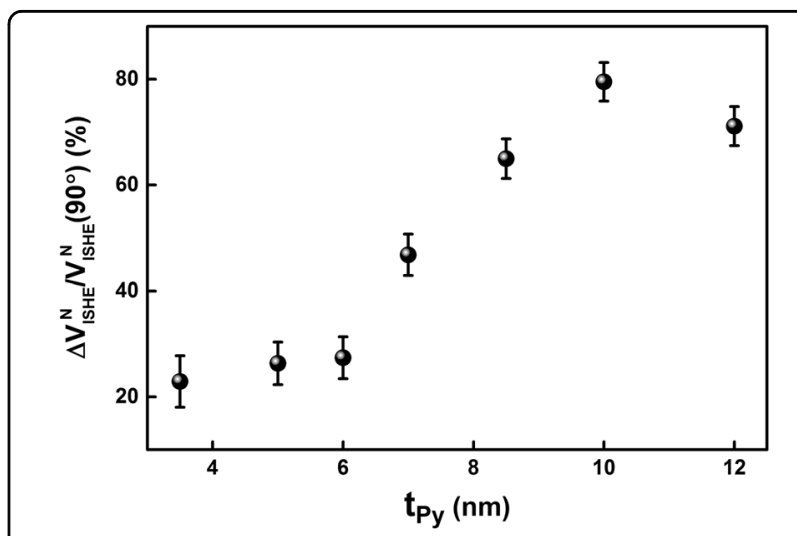

Fig. 3 Magnitude of ISHE modulated by magnetization orientation. The ratio of $V_{\text {ISHE }}$ difference at $\theta=0^{\circ}$ and $\theta=90^{\circ}, \operatorname{Ra}\left(V_{\mathrm{ISHE}}\right)=\frac{\Delta V_{\mathrm{ISHE}}}{V_{\mathrm{ISHE}}\left(90^{\circ}\right)^{\prime}}$ varies at different Py thicknesses. The error bars represent the 68\% confidence level $( \pm S D)$.

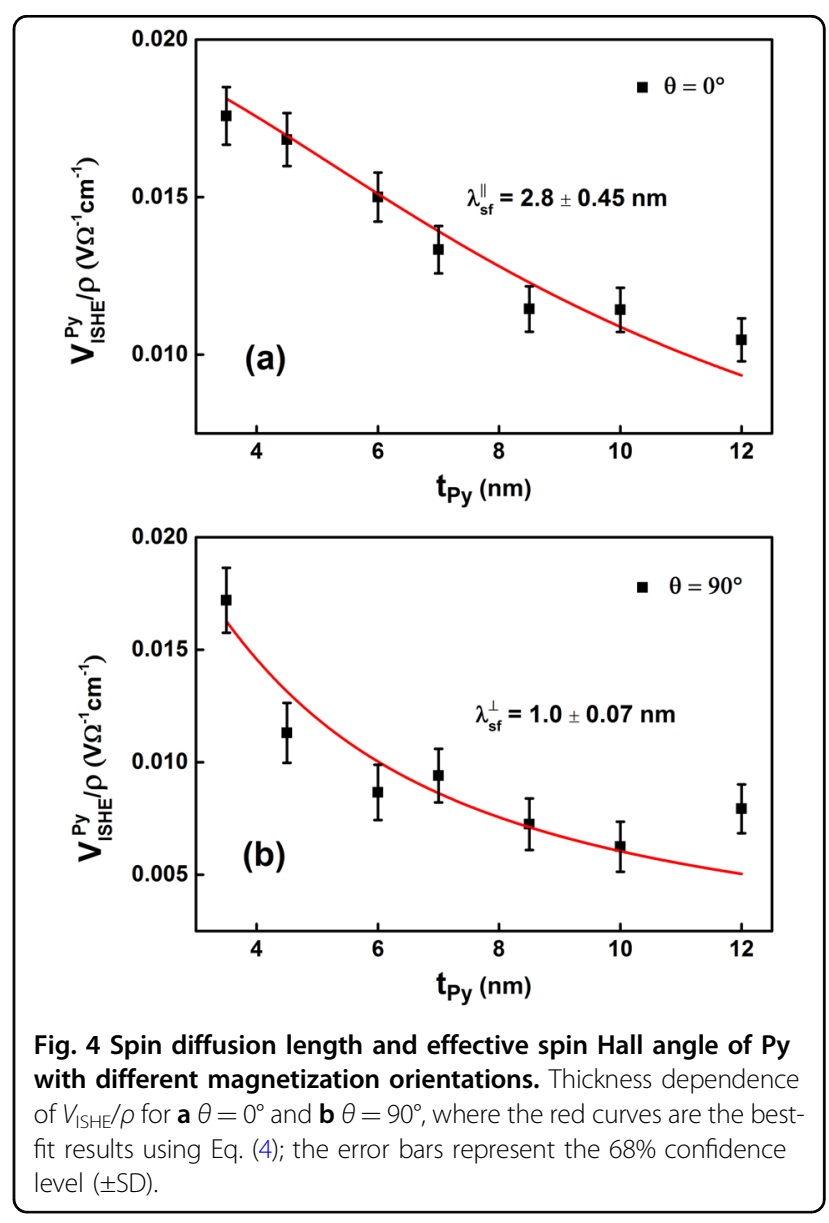

layers (for more details, see the Supplementary Information Section C). Figure 4a, b presents the dependence of $V_{\mathrm{ISHE}}^{\mathrm{Py}} / \rho$ on Py thickness at $\theta=0^{\circ}$ and $\theta=90^{\circ}$. According to the open-circuit model of the ISHE in metals, the thermal voltage in Py caused by the ISHE can 
be described by ${ }^{12}$

$$
\frac{V_{\mathrm{ISHE}}^{\mathrm{Py}}}{\rho}=J_{\mathrm{s}}(0) \theta_{\mathrm{SH}} L \frac{\lambda_{\mathrm{sf}}}{t_{\mathrm{Py}}} \tanh \left(\frac{t_{\mathrm{Py}}}{2 \lambda_{\mathrm{sf}}}\right),
$$

where $\rho$ is the resistivity of the Py layer, $J_{\mathrm{s}}(0)$ is the spin current density at the interface and is proportional to the temperature gradient $\nabla T, \theta_{\mathrm{SH}}$ is the spin Hall angle, $\lambda_{\mathrm{sf}}$ is the spin diffusion length and $L \approx 5.6 \mathrm{~mm}$ is the length of the Py film. The experimental results are fitted with Eq. (4) for $\theta=0^{\circ}$ and $\theta=90^{\circ}$. The fitting results revealed that both $\lambda_{\mathrm{sf}}$ and the effective spin-to-charge conversion efficiency $\theta_{\mathrm{SH}}^{\text {eff }}=J_{\mathrm{s}}(0) \theta_{\mathrm{SH}}$ in Py are strongly $\theta$ dependent. Remarkably, the ratio of the effective spin Hall angle $\theta_{\mathrm{SH}}^{\text {eff }}(\perp) / \theta_{\mathrm{SH}}^{\text {eff }}(\|)$ is as large as 1.5 , where $\perp$ and $\|$ represent $\theta=90^{\circ}$ and $\theta=0^{\circ}$, respectively. Moreover, $\lambda_{\mathrm{sf}}^{\|}=2.8 \pm$ $0.5 \mathrm{~nm}$ at $\theta=0^{\circ}$, which is consistent with the results for Py reported previously ${ }^{12,16}$. In contrast, $\lambda_{\mathrm{sf}}^{\perp}=1.0 \pm 0.1 \mathrm{~nm}$ at $\theta=90^{\circ}$, much smaller than the value for $\theta=0^{\circ}$.

\section{Discussion}

To understand these $\theta$-dependent spin transport phenomena, the dynamics of spin accumulation at the interfaces and spin current transport in Py should be taken into account. Assuming that the bilayer Py/IrMn is a perfect spin sink, $J_{\mathrm{s}}(0)$ can be expressed as ${ }^{36,37}$

$$
\begin{aligned}
\vec{J}_{\mathrm{s}}(0)= & G_{\mathrm{r}}^{\uparrow \downarrow} \vec{M}_{\mathrm{Py}} \times \vec{\mu}_{\mathrm{s}, \mathrm{Cu}} \times \vec{M}_{\mathrm{Py}}+G_{\mathrm{i}}^{\uparrow} \vec{\mu}_{\mathrm{s}, \mathrm{Cu}} \\
& \times \vec{M}_{\mathrm{Py}}+G^{\uparrow \uparrow} \vec{M}_{\mathrm{Py}} \cdot\left(\vec{\mu}_{\mathrm{s}, \mathrm{Cu}} \cdot \vec{M}_{\mathrm{Py}}\right),
\end{aligned}
$$

where $G_{r}^{\uparrow \downarrow}$ and $G_{i}^{\uparrow \downarrow}$ are respectively the real part and imaginary part of the transverse spin mixing conductance for the $\mathrm{Cu} / \mathrm{Py}$ interface, $G^{\Uparrow \uparrow}$ is the longitudinal spin conductance, and $\vec{\mu}_{\mathrm{s}, \mathrm{Cu}}$ is the spin accumulation vector in the $\mathrm{Cu}$ layer, which also represents the spin polarization direction of the spin current. The first two terms on the right are the spin current with polarization orthogonal to $\vec{M}_{\text {Py }}$, and the last term on the right is the spin current with polarization parallel to $\vec{M}_{\mathrm{Py}}$. All the spin currents mentioned above flow perpendicular to interfaces. According to the recent models in $\operatorname{LSSE}^{38,39}$, the thermal spin current originates from the bulk effect, namely, FMI generates nonequilibrium thermal magnons under a temperature gradient, which leads to $\vec{\mu}_{\mathrm{s}, \mathrm{Cu}}$ parallel to $\vec{M}_{\text {YIG }}$ in our case. Under the same $\nabla T$ and Cu thickness, $\vec{J}_{\mathrm{s}}(0)$ is determined by the spin-dependent mixing conductance and $\theta$. Considering that $G_{\mathrm{i}}^{\uparrow \downarrow} \ll G_{\mathrm{r}}^{\uparrow \downarrow}$, in the case of $\theta=0^{\circ}$ and $\theta=90^{\circ}$, the corresponding $\vec{J}_{s}(0)$ is determined solely by $G^{\uparrow \uparrow}$ and $G_{r}^{\uparrow \downarrow}$. As shown in the spin Hall magnetoresistance studies in metallic bilayers ${ }^{40}, G^{\uparrow \uparrow}$ is smaller than $G_{r}^{\uparrow \downarrow}$ at room temperature. This should partially contribute to the experimental observation that $\theta_{\mathrm{SH}}^{\text {eff }}(\perp)$ is larger than $\theta_{\mathrm{SH}}^{\text {eff }}(\|)$. In addition, with the discovery of magnetization-dependent spin-to-charge conversation effect in $\mathrm{FMM}^{18,41}, \theta_{\mathrm{SH}}$ might also depend on $\theta$ considerably and contribute to the observed larger $\theta_{\mathrm{SH}}^{\text {eff }}(\perp)$ too. In the lack of a systematic study on the magnetization orientation related $\theta_{\mathrm{SH}}$ in FMM, we phenomenologically attribute the differences in $\theta_{\mathrm{SH}}^{\text {eff }}$ at different $\theta$ to the different spin current injection efficiencies across the interface.

Except for the spin-dependent scattering at the interface, spin diffusion of the spin accumulation $\left(\vec{\mu}_{\mathrm{s}}\right)$ parallel (denoted as $\vec{\mu}_{\mathrm{s}}^{\|}$) and transverse (denoted as $\vec{\mu}_{\mathrm{s}}^{\perp}$ ) to $\vec{M}_{\text {Py }}$ must be considered separately ${ }^{42}$ to explain the change of $\lambda_{\text {sf }}$ with $\theta$, which can be written as follows:

$$
\begin{aligned}
& \frac{\partial^{2} \vec{\mu}_{\mathrm{s}}^{\|}}{\partial z^{2}}-\frac{\vec{\mu}_{\mathrm{s}}^{\|}}{\lambda_{\mathrm{sf}}^{2}\left(1-\beta^{2}\right)}=0, \\
& \frac{\partial^{2} \vec{\mu}_{\mathrm{s}}^{\perp}}{\partial z^{2}}-\frac{\vec{\mu}_{\mathrm{s}}^{\perp}}{\lambda_{\mathrm{sf}}^{2}}-\frac{\vec{\mu}_{\mathrm{s}}^{\perp} \times \vec{M}_{\mathrm{Py}}}{\lambda_{J}}=0 .
\end{aligned}
$$

When $\theta=0^{\circ}, \vec{\mu}_{\mathrm{s}}$ is parallel to $\vec{M}_{\mathrm{Py}}$; as mentioned above, the transport of $\vec{\mu}_{\mathrm{s}}$ described by Eq. (6) is analogous to the spin diffusion in the normal metal except for a coefficient modification by the spin polarization parameter $(\beta)$ inherent to Py. As a result, $\mu_{\mathrm{s}} \propto \exp \left(-\frac{z}{\lambda_{\mathrm{sf}} \sqrt{1-\beta^{2}}}\right)$. However, when the direction of $\vec{\mu}_{\mathrm{s}}$ is turned from parallel to perpendicular to $\vec{M}_{\mathrm{Py}}$, the mechanism of the spatial variation of transverse spin accumulation is governed mainly by the precession induced by the exchange field in Py according to a theoretic model described by Eq. (7). As a consequence, $\mu_{\mathrm{s}} \propto \exp \left(-\frac{z}{\lambda_{J}}\right)$, where the characteristic exchange length $\left(\lambda_{J}\right)$ is commonly viewed to be smaller than the spin diffusion length $\left(\lambda_{\mathrm{sf}}\right)$. Thus, the decay of the transverse spin accumulation in ferromagnetic metals is more rapid than its longitudinal counterpart. It should be pointed out that $\lambda_{\mathrm{sf}}^{\perp}$ calculated from Eq. (4) actually corresponds to the quantity of $\lambda_{F}$, rather than the common spin diffusion length $\lambda_{\text {sf }}$. The latter can be acquired from the longitudinal spin diffusion length $\lambda_{\mathrm{sf}}^{\|}$in our experiments according to $\lambda_{\mathrm{sf}}=\frac{\lambda_{\mathrm{sf}}^{\|}}{\sqrt{1-\beta^{2}}}$. We would like to emphasize that $\lambda_{\mathrm{sf}}^{\perp}$ or $\lambda_{J}$ in our result cannot be taken as the transverse spin coherence length $\lambda_{\phi} \sim \frac{2 \pi}{\left|k_{\mathrm{f}}^{\dagger}-k_{\mathrm{f}}^{\downarrow}\right|} 43$, in which $k_{\mathrm{f}}^{\uparrow(\downarrow)}$ represents the majority (minority) Fermi wave vectors. In addition, $\lambda_{\phi}$ is widely studied in FMR measurements of spin pumping ${ }^{23,24}$, and it is often characterized by the enhancement of the damping factor in a 
spin valve structure compared with the single FM layer. In the present thermal ISHE measurement, the ISHE voltage is the direct detection of the spin current injected into the metals, and $\lambda_{\text {sf }}^{\perp}$ or $\lambda_{J}$ is the characteristic decay length for the transverse spin accumulation governed by the exchange interaction in ferromagnetic metals ${ }^{42}$. However, $\lambda_{\phi}$ is irrelevant to the spin accumulation and diffusion in ferromagnetic metals and sets a length scale of a power law that contrasts with the exponential suppression for transverse spin accumulation ${ }^{37,44,45}$. Our results provide strong evidence that the spin relaxation of the pure current in Py is anisotropic and that the relaxation mechanisms between longitudinal and transverse spin accumulation in ferromagnetic metals are quite different. Finally, we would like to emphasize that the introduction of the antiferromagnetic IrMn layer helps to effectively absorb the spin current and to fix the Py moment, which greatly facilitates the exploration of spin orientation-dependent transport of pure spin current in a ferromagnetic metal.

In conclusion, by using the $\mathrm{YIG} / \mathrm{Cu} / \mathrm{Py} / \mathrm{IrMn}$ spin valve heterostructure, we observed that the diffusion of the pure spin current and the inverse spin Hall effect in Permalloy are dependent on its magnetization. We have revealed that the spin orientation-relevant injection efficiency across the interface and spin relaxation of pure spin current in Py are the main reasons for these anisotropic spin transport phenomena. The present results provide a unified understanding of the impact of the magnetization or exchange interaction on the pure spin current transport in ferromagnetic metals.

\section{Acknowledgements}

This work was supported by the National Key Research and Development Program of China under Grant No. 2016YFA0300804, the National Basic Research Program of China under Grant No. 2015CB921403, the National Natural Science Foundation of China under Grant Nos. 11674379 and 51431009, and the Key Science and Technology Research Program of Beijing Polytechnic under Grant No. 2019Z002-006-KXB.

\section{Author details}

'Beijing National Laboratory for Condensed Matter, Institute of Physics, Chinese Academy of Sciences, Beijing 100190, People's Republic of China. ${ }^{2}$ School of Physical Sciences, University of Chinese Academy of Sciences, Beijing 100049, People's Republic of China. ${ }^{3}$ Beijing Polytechnic, Beijing 100176, People's Republic of China

\section{Conflict of interest}

The authors declare that they have no conflict of interest.

\section{Publisher's note}

Springer Nature remains neutral with regard to jurisdictional claims in published maps and institutional affiliations.

Supplementary information is available for this paper at https://doi.org/ 10.1038/s41427-019-0191-1.

Received: 17 July 2019 Revised: 5 November 2019 Accepted: 19 November 2019.

Published online: 17 January 2020

\section{References}

1. Kajiwara, Y. et al. Transmission of electrical signals by spin-wave interconversion in a magnetic insulator. Nature 464, 262-266 (2010).

2. Uchida, K. et al. Spin Seebeck insulator. Nat. Mater. 9, 894-897 (2010).

3. Uchida, K.-i. et al. Observation of longitudinal spin-Seebeck effect in magnetic insulators. Appl. Phys. Lett. 97, 172505 (2010).

4. Qu, D. et al. Intrinsic spin Seebeck effect in Au/YIG. Phys. Rev. Lett. 110, 067206 (2013).

5. Kikkawa, T. et al. Longitudinal spin Seebeck effect free from the proximity Nernst effect. Phys. Rev. Lett. 110, 067207 (2013).

6. Meier, D. et al. Longitudinal spin Seebeck effect contribution in transverse spin Seebeck effect experiments in Pt/YIG and Pt/NFO. Nat. Commun. 6, 8211 (2015).

7. Ideue, T. et al. Effect of lattice geometry on magnon Hall effect in ferromagnetic insulators. Phys. Rev. B. 85, 134411 (2012).

8. Onose, Y. et al. Observation of the magnon Hall effect. Science 329, 297-299 (2010).

9. Madon, B. et al. Anomalous and planar Righi-Leduc effects in $\mathrm{Ni}_{80} \mathrm{Fe}_{20}$ ferromagnets. Phys. Rev. B. 94, 144423 (2016).

10. Wegrowe, J. E., Drouhin, H. J. \& Lacour, D. Anisotropic magnetothermal transport and spin Seebeck effect. Phys. Rev. B. 89, 094409 (2014).

11. Chen, Y. J. \& Huang, S. Y. Absence of the thermal Hall effect in anomalous Nernst and spin Seebeck effects. Phys. Rev. Lett. 117, 247201 (2016).

12. Miao, B. F. et al. Inverse spin Hall effect in a ferromagnetic metal. Phys. Rev. Lett. 111, 066602 (2013).

13. Zheng, X. L. et al. Large inverse spin Hall effect in Co-Pt spin-valve heterostructures. Phys. Rev. Appl. 7, 044003 (2017).

14. Seki, T. et al. Observation of inverse spin Hall effect in ferromagnetic FePt alloys using spin Seebeck effect. Appl. Phys. Lett. 107, 092401 (2015).

15. Kikkawa, T. et al. Separation of longitudinal spin Seebeck effect from anomalous Nernst effect: determination of origin of transverse thermoelectric voltage in metal/insulator junctions. Phys. Rev. B. 88, 214403 (2013).

16. Wang, $H$. et al. Spin current and inverse spin Hall effect in ferromagnetic metals probed by $\mathrm{Y}_{3} \mathrm{Fe}_{5} \mathrm{O}_{12}$-based spin pumping. Appl. Phys. Lett. 104, 202405 (2014).

17. Hyde, P. et al. Electrical detection of direct and alternating spin current injected from a ferromagnetic insulator into a ferromagnetic metal. Phys. Rev. B. 89, 180404 (2014).

18. Das, K. S. et al. Spin injection and detection via the anomalous spin Hall effect of a ferromagnetic metal. Phys. Rev. B. 96, 220408 (2017).

19. Omori, Y. et al. Relation between spin Hall effect and anomalous Hall effect in 3d ferromagnetic metals. Phys. Rev. B. 99, 014403 (2019).

20. Cramer, J. et al. Orientation-dependent direct and inverse spin Hall effects in $\mathrm{Co}_{60} \mathrm{Fe}_{20} \mathrm{~B}_{20}$. Phys. Rev. B. 99, 104414 (2019).

21. Steenwyk, S. D. et al. Perpendicular-current exchange-biased spin-valve evidence for a short spin-diffusion lenght in permalloy. J. Magn. Magn. Mater. 170, L1-L6 (1997)

22. Yang, Q. et al. Spin flip diffusion length and giant magnetoresistance at low temperatures. Phys. Rev. Lett. 72, 3274-3277 (1994).

23. Taniguchi, T. et al. Determination of penetration depth of transverse spin current in ferromagnetic metals by spin pumping. Appl. Phys. Express 1, 031302 (2008).

24. Ghosh, A. et al. Penetration depth of transverse spin current in ultrathin ferromagnets. Phys. Rev. Lett. 109, 127202 (2012).

25. Tian, D. et al. Manipulation of pure spin current in ferromagnetic metals independent of magnetization. Phys. Rev. B. 94, 020403 (2016).

26. Li, Y., Cao, W. \& Bailey, W. E. Characterization of spin relaxation anisotropy in Co using spin pumping. Phys. Rev. B. 94, 174439 (2016).

27. Freimuth, F., Blugel, S. \& Mokrousov, Y. Anisotropic spin Hall effect from first principles. Phys. Rev. Lett. 105, 246602 (2010).

28. Kimata, M. et al. Magnetic and magnetic inverse spin Hall effects in a non collinear antiferromagnet. Nature 565, 627-630 (2019).

29. Zhang, W. et al. Giant facet-dependent spin-orbit torque and spin Hall conductivity in the triangular antiferromagnet IrMn 3 . Sci. Adv. 2, e1600759 (2016).

30. Baker, A. A. et al. Anisotropic absorption of pure spin currents. Phys. Rev. Lett. 116, 047201 (2016)

31. Bai, H. et al. Characterization of YIG thin films and vacuum annealing effect by polarized neutron reflectometry and magnetotransport measurements. Appl. Phys. Lett. 115, 182401 (2019).

32. Li, G. et al. Tunable perpendicular magnetic anisotropy in epitaxial $\mathrm{Y}_{3} \mathrm{Fe}_{5} \mathrm{O}_{12}$ films. APL Mater. 7, 041104 (2019). 
33. Tshitoyan, $\mathrm{V}$. et al. Electrical manipulation of ferromagnetic NiFe by antiferromagnetic IrMn. Phys. Rev. B. 92, 214406 (2015).

34. Saglam, $H$. et al. Independence of spin-orbit torques from the exchange bias direction in $\mathrm{Ni}_{81} \mathrm{Fe}_{19} / \mathrm{lrMn}$ bilayers. Phys. Rev. B. 98, 094407 (2018).

35. Lu, Y. M. et al. Hybrid magnetoresistance in the proximity of a ferromagnet. Phys. Rev. B. 87, 220409 (2013).

36. Tserkovnyak, Y., Brataas, A. \& Bauer, G. E. W. Dynamic stiffness of spin valves. Phys. Rev. B. 67, 140404 (2003).

37. Tserkovnyak, Y. et al. Nonlocal magnetization dynamics in ferromagnetic heterostructures. Rev. Mod. Phys. 77, 1375-1421 (2005).

38. Rezende, S. M. et al. Magnon spin-current theory for the longitudinal spinSeebeck effect. Phys. Rev. B. 89, 014416 (2014).

39. Ritzmann, U., Hinzke, D. \& Nowak, U. Propagation of thermally induced magnonic spin currents. Phys. Rev. B. 89, 024409 (2014).
40. Kim, J. et al. Spin Hall magnetoresistance in metallic bilayers. Phys. Rev. Lett. 116, 097201 (2016)

41. Cramer, J. et al. Magnon detection using a ferroic collinear multilayer spin valve. Nat. Commun. 9, 1089 (2018)

42. Zhang, S., Levy, P. M. \& Fert, A. Mechanisms of spin-polarized current-driven magnetization switching. Phys. Rev. Lett. 88, 236601 (2002).

43. Zwierzycki, M. et al. First-principles study of magnetization relaxation enhancement and spin transfer in thin magnetic films. Phys. Rev. B. 71, 064420 (2005).

44. Zhang, J. et al. Identification of transverse spin currents in noncollinear magnetic structures. Phys. Rev. Lett. 93, 256602 (2004).

45. Stiles, M. D. \& Zangwill, A. Anatomy of spin-transfer torque. Phys. Rev. B. 66 014407 (2002). 